\title{
Temperature and orientation dependence of kinetic roughening during homoepitaxy: A quantitative x-ray-scattering study of $\mathbf{A g}$
}

\author{
W. C. Elliott and P. F. Miceli \\ Department of Physics and Astronomy, University of Missouri-Columbia, Columbia, Missouri 65211 \\ T. Tse and P. W. Stephens \\ Department of Physics, State University of New York, Stony Brook, New York 11794
}

(Received 13 August 1996)

\begin{abstract}
Kinetic roughening during homoepitaxial growth was studied for $\operatorname{Ag}(111)$ and $\operatorname{Ag}(001)$. For $\operatorname{Ag}(111)$, from 150 to $500 \mathrm{~K}$, the rms roughness exhibits a power law, $\sigma \propto t^{\beta}$ over nearly three decades in thickness. $\beta \approx \frac{1}{2}$ at low temperatures, and there is an abrupt transition to smaller values above $300 \mathrm{~K}$. In contrast, $\operatorname{Ag}(001)$ exhibits layer-by-layer growth with a significantly smaller $\beta$. These results are the first to establish the evolution of surface roughness quantitatively for a broad thickness and temperature range, as well as for the case where growth kinetics are dominated by a step-ledge diffusion barrier. [S0163-1829(96)00247-0]
\end{abstract}

\section{INTRODUCTION}

Kinetic roughening, where an initially smooth surface progressively roughens as atoms deposit from a vapor, is a subject of considerable fundamental interest, and it is important to technological applications which rely on the ability to control the surface morphology of thin films. Many theoretical studies can be found in the literature, ${ }^{1}$ which propose a scaling behavior for a growing rough surface; however, there are comparatively few experimental studies ${ }^{2}$ which critically test the theories, nor do these adequately address the microscopic origin of roughening. The importance of microscopic mechanisms has been recognized in recent theoretical ${ }^{3-9}$ and experimental $^{10-14}$ work. It is, therefore, useful to examine growth on the simplest crystalline systems, in order to establish the fundamental behavior of kinetic roughening.

The time evolution of this type of roughened surface derives from two sources: a noisy (thermal) flux of impinging atoms and the relaxation physics that governs the rearrangement of atoms once they arrive at the surface. Consequently, it is essential ${ }^{15}$ to consider the specific relaxation mechanisms that are relevant to growth at real crystalline surfaces: nucleation, surface diffusion, and diffusion over crystalline surface step ledges. The importance of such mechanisms is highlighted by the well-known growth modes observed during homoepitaxy-step flow, layer by layer, and multilayer-which are qualitative manifestations of these relaxation mechanisms. At present, a quantitative description of kinetic roughening that interrelates these qualitative phenomena has not been adequately established.

A key issue concerns the formation of mounds during growth, and a number of recent experiments on singular orientation surfaces ${ }^{10-13}$ have revealed such growth instabilities. This situation arises from a diffusion bias, ${ }^{3}$ as can be demonstrated by considering the stability during deposition of a two-dimensional island having a single atomic-layer height. If incoming atoms deposited atop the island experience a barrier to diffusion over the island's edge (the Ehrlich-Schwoebel barrier), then the next atomic layer may nucleate before the previous layer completes, thereby leading to multilayer (three-dimensional) growth and the eventual appearance of mounds. If, however, this step-ledge barrier is negligibly small, then the atomic layer completes before the next one nucleates so that the growth is layer by layer (LBL), yielding a relatively smooth surface. A characteristic signature of LBL growth comes from reflection-high-energy electron diffraction (RHEED) measured in situ, where oscillations of the out-of-phase reflected intensity (RHEED oscillations) occur with the period needed to complete a monolayer. In practice the intensity tends to weaken with successive oscillations due to the step-ledge barrier. ${ }^{16}$ Therefore, the step-ledge barrier is centrally important in determining the growth morphology and any discussion of power-law exponents, if these are observable at all, must consider such microscopic kinetics which will certainly vary with the temperature as well as the orientation of the crystal surface. In fact, a measurement of the temperature dependence would seem to be a prerequisite for establishing a particular growth model.

In this paper, we report the temperature dependence of the surface roughness during homoepitaxy on $\operatorname{Ag}(111)$ and $\mathrm{Ag}(001)$, where the two orientations provide opposite limits of step-ledge barrier effects. For $\mathrm{Ag}(111)$, it is known that RHEED oscillations are not present ${ }^{17}$ at any temperature, indicating a large step-ledge barrier, ${ }^{18,19}$ and this represents the important limiting case where a single mechanism dominates the growth kinetics. $\mathrm{Ag}(001)$, in contrast, exhibits RHEED oscillations $^{20,21}$ at low temperature, suggesting a relatively small step-ledge barrier. Our results for $\operatorname{Ag}(111)$ show, remarkably, that over nearly three decades in deposited thickness and over the entire temperature range examined $(150-500 \mathrm{~K})$ the rms surface roughness is well described by a power law, $\sigma \propto t^{\beta}$. We observe a transition in the exponent $\beta$, where $\beta \approx \frac{1}{2}$ at low temperature and rapidly decreases above $300 \mathrm{~K}$ - this is concomitant with a steady decrease in the roughness magnitude. For $\operatorname{Ag}(001), \beta$ also varies with temperature, but exhibits values that are significantly smaller than what is observed on $\operatorname{Ag}(111)$ at a given temperature. We believe these results are roughly consistent with recent computer simulations, ${ }^{6,9,27,28}$ although the quantitative 


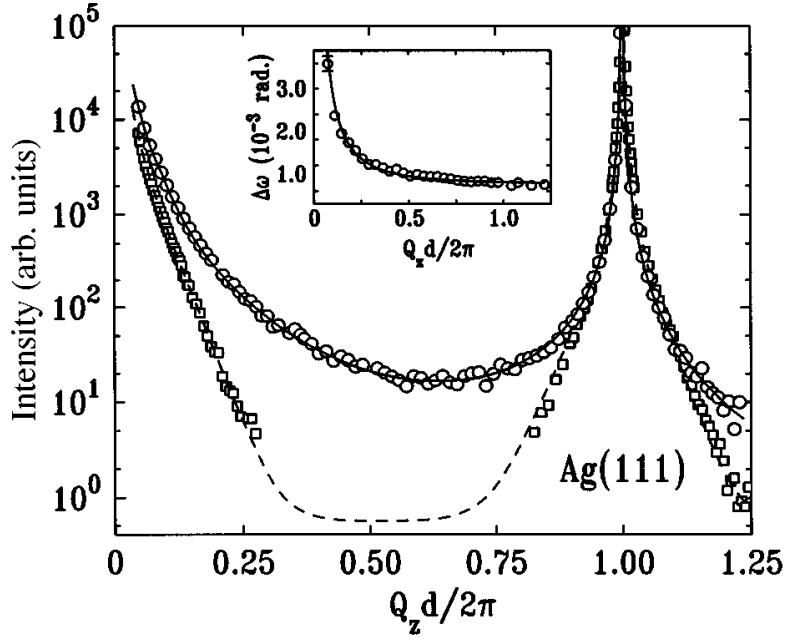

FIG. 1. The specular x-ray reflectivity is shown for a smooth $\operatorname{Ag}(111)$ starting surface (circles) and a rough surface (squares) with $3.2 \AA$ deposited at $200 \mathrm{~K}$. The solid and dashed curves are a least-squares fit to Eq. (1) with geometrical corrections included. The inset shows the wave-vector dependence of the transverse angular width of the specular peak; this gives a typical length of $\sim 9000 \AA$ for flat $\operatorname{Ag}(111)$ facets.

information (roughness magnitude as well as exponent) available from our experiments potentially affords a rigorous test of growth models.

\section{EXPERIMENT}

The experiments used an ultrahigh vacuum chamber (ambient, low $10^{-10}$ Torr) located on the SUNY X3B2 beamline at the National Synchrotron Light Source. Ag was deposited from a thermal oven, and the deposition flux was interrupted to allow in situ $\mathrm{x}$-ray reflectivity (1.1379 ^) measurements. Substrates were mechanically polished (miscut $\sim 0.1^{\circ}$ ) followed by repeated cycles of annealing $\left(700-750{ }^{\circ} \mathrm{C}\right)$ and Ar-ion sputtering. No surface impurities were detectable by Auger analysis. A highly smooth starting surface was prepared before each deposition series by repeated sputter/ anneal cycles. The specular $\mathrm{x}$-ray reflectivity data, with the diffuse scattering subtracted, were analyzed according to 22

$$
R \propto|f(Q)|^{2} \frac{\exp \left(-4 \frac{\sigma^{2}}{d^{2}} \sin ^{2}\left(\frac{Q_{z} d}{2}\right)\right)}{Q_{z}^{2} \sin ^{2}\left(\frac{Q_{z} d}{2}\right)},
$$

which allowed the rms surface roughness $\sigma$ to be obtained. Here $Q_{z}=4 \pi / \lambda \sin \theta$ is the wave-vector transfer perpendicular to the surface, $\lambda$ is the wavelength, $\theta$ is the angle of specular reflection, $d$ is the atomic plane spacing normal to the surface, and $f(Q)$ is the atomic form factor.

\section{RESULTS}

Figure 1 shows the specular reflectivity for an as-prepared $\operatorname{Ag}(111)$ surface where the solid curve is a least-squares fit to Eq. (1). We find $\sigma<0.5 \AA$, and such a small roughness can

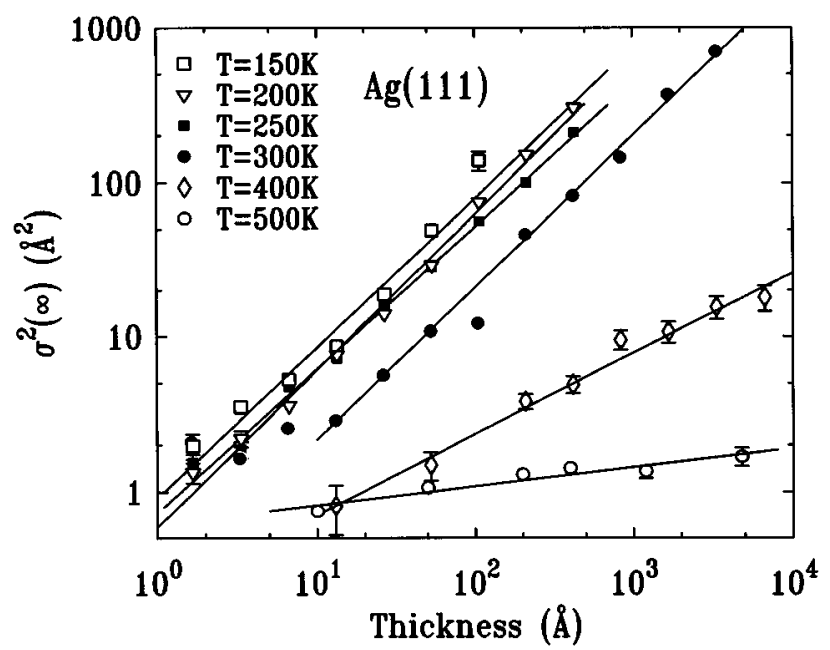

FIG. 2. The mean-square roughness of the $\operatorname{Ag}(111)$ surface, measured by $\mathrm{x}$-ray reflectivity, is shown as a function of the deposited Ag thickness at six different temperatures. The solid lines are obtained from a fit to $\sigma^{2} \propto t^{2 \beta}$.

only be detected by measuring the reflectivity over this large wave-vector range. The roughness is obtained over a lateral area corresponding to the correlation length $L$ of the surface, which was determined from the transverse angular broadening of the specular peak, ${ }^{22}$ shown in the inset to Fig. 1 . We obtain $L \sim 9000 \AA$, and this implies that our experiments take place on large, flat $\operatorname{Ag}(111)$ facets whose roughness is just a fraction of an $\AA$. Note that high-temperature annealing is responsible for the formation of the facets, and these possess terrace sizes that are much larger than what would be expected from the miscut of the crystal; in fact, we can directly observe the increase in $L$ as the sample is annealed. Such surfaces are routinely achieved. As we will demonstrate, the starting surface quality is very important in kinetic roughening studies.

The evolution of $\sigma^{2}$ with film thickness is shown in Fig. 2 for different temperatures. The deposition rate was either 7 or $25 \AA / \mathrm{min}$, and the results did not depend on the choice of rate. As shown by the square data points in Fig. 1, the reflectivity measured at grazing angles and near the Bragg reflection yield the same roughness, indicating that the deposited atoms enter the epitaxial lattice sites rather than form a polycrystalline film. We also confirmed the absence of annealing effects due to the interruption of the deposition flux. ${ }^{22}$ This is consistent with a low rate for atoms to detach from islands.

As shown in Fig. 2, the roughness evolution at each temperature is well described by a power law. Figure 3 shows that, although the roughness decreases continuously with increasing temperature, $\beta \approx \frac{1}{2}$ is constant at low temperature, and decreases rapidly above $300 \mathrm{~K}$. We performed these measurements many times, and carefully examined their reproducibility; consequently, the error bars in Fig. 3(b) represent the bounds of experimental reproducibility in obtaining $\beta$ rather than statistical uncertainty (which would be smaller).

A qualitatively different growth behavior occurs for growth on the $\operatorname{Ag}(001)$ orientation. As shown in Fig. 4, the intensity near the anti-Bragg position exhibits pronounced 


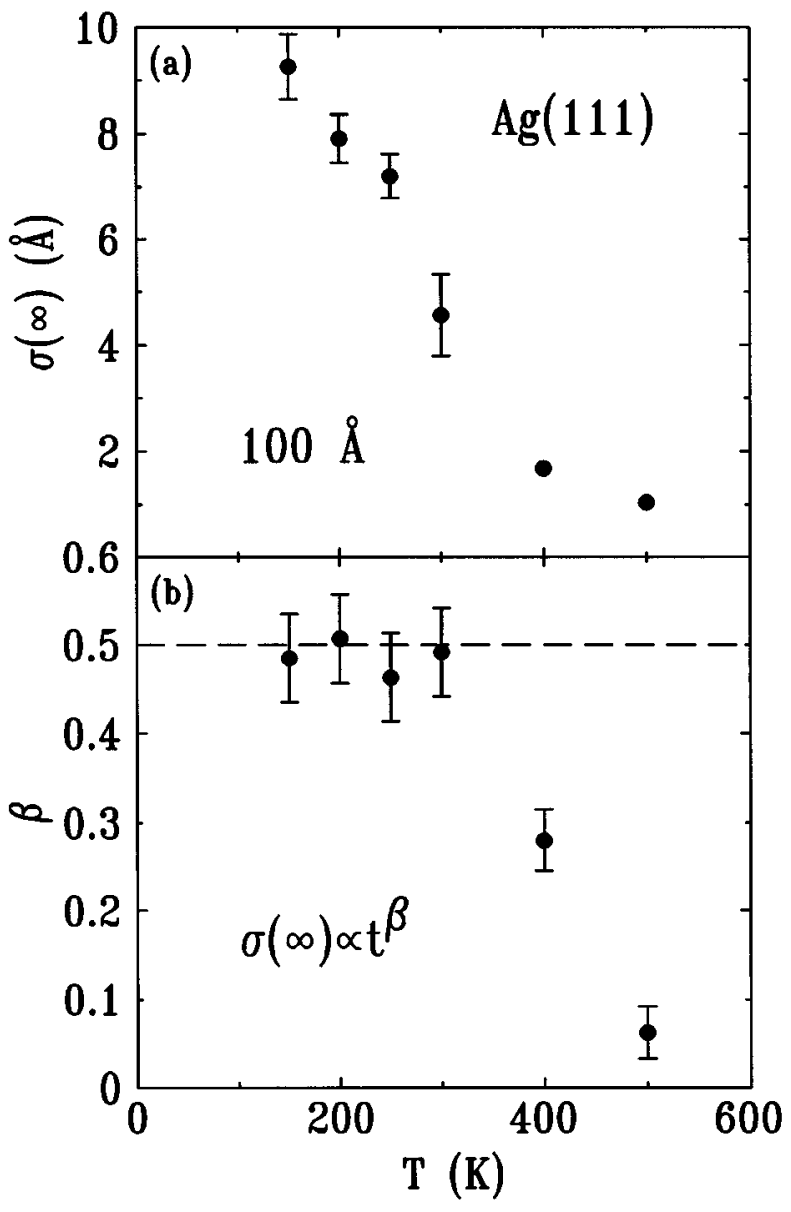

FIG. 3. The temperature dependences of (a) $\sigma$ at $100 \AA$ of deposited $\mathrm{Ag}$ and (b) $\beta$ are obtained from the data in Fig. 2. There is an abrupt transition in $\beta$ above $300 \mathrm{~K}$. Although $\beta \approx \frac{1}{2}$ is not changing below $300 \mathrm{~K}$, the roughness magnitude decreases steadily with increasing temperature.

oscillations during deposition, indicating LBL growth. These become highly damped at low temperature due to the rapidly increasing roughness. At the highest temperature growth proceeds by step flow, as the oscillations are absent and the intensity decreases only slightly. The thickness dependence of the roughness is shown in Fig. 5, from which we find $\beta=0.28 \pm 0.03$ at $200 \mathrm{~K}$ and $\beta=0.17 \pm 0.02$ at $300 \mathrm{~K}$. Thus, for a given temperature the exponents are much smaller for $\operatorname{Ag}(001)$ as compared to $\operatorname{Ag}(111)$, although we were not able to check if $\beta$ approaches $\frac{1}{2}$ at lower temperature. At $300 \mathrm{~K}$ there is a dramatic departure from a power law for large film thicknesses; this result is entirely reproducible, and it is evidently intrinsic to the growth behavior. We are not aware of models which predict such a delayed, precipitous increase of the roughness.

\section{DISCUSSION}

We now discuss the exponent $\beta=\frac{1}{2}$. First, it is important to recognize that there is significant mobility on a flat $\mathrm{Ag}(111)$ terrace at low temperature, ${ }^{19}$ but that the additional energy barrier to diffusion over a step ledge on $\mathrm{Ag}(111)$ is quite large, ${ }^{18,19}$ being slightly greater than the energy barrier

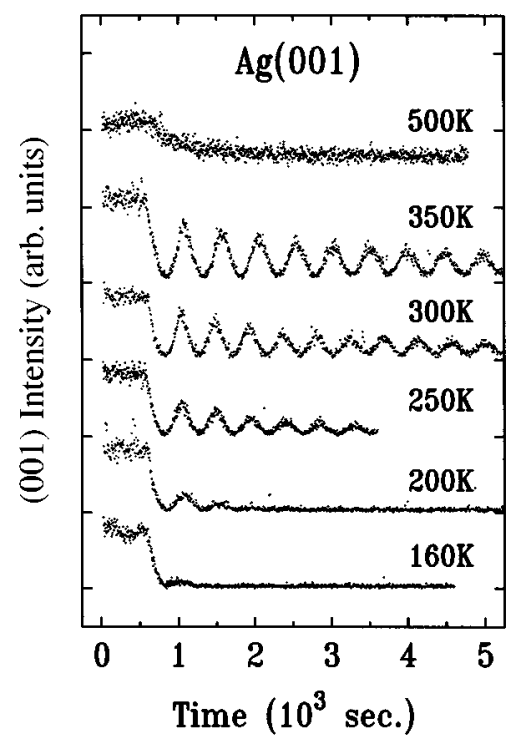

FIG. 4. The specularly reflected intensity near the anti-Bragg position for $\operatorname{Ag}(001)$ was measured during growth (deposition rate $\approx 0.3 \AA / \mathrm{min}) . \mathrm{Ag}(001)$ exhibits layer-by-layer growth, and each period of intensity oscillation corresponds to one atomic layer deposited. The oscillations are highly damped at low temperature, whereas step-flow growth occurs at $500 \mathrm{~K}$, where the oscillations are absent and the intensity decreases only slightly.

for surface diffusion. This leads to multilayer growth, with the formation of mounds, ${ }^{18,23,24}$ and the complete absence ${ }^{17}$ of intensity oscillations such as those observed for $\mathrm{Ag}(001)$ in Fig. 4. A number of models which limit lateral relaxation have yielded $\beta=\frac{1}{2}, 1,15,25$ and this occurs when there is no interlayer hopping, even in the presence of intralayer hopping. ${ }^{25}$ More extensive Monte Carlo simulations, ${ }^{6}$ which include several relevant microscopic kinetic mechanisms as well as lead to mound formation, also give $\beta=\frac{1}{2}$ when the step-ledge barrier is insurmountable. Thus, there is consider-

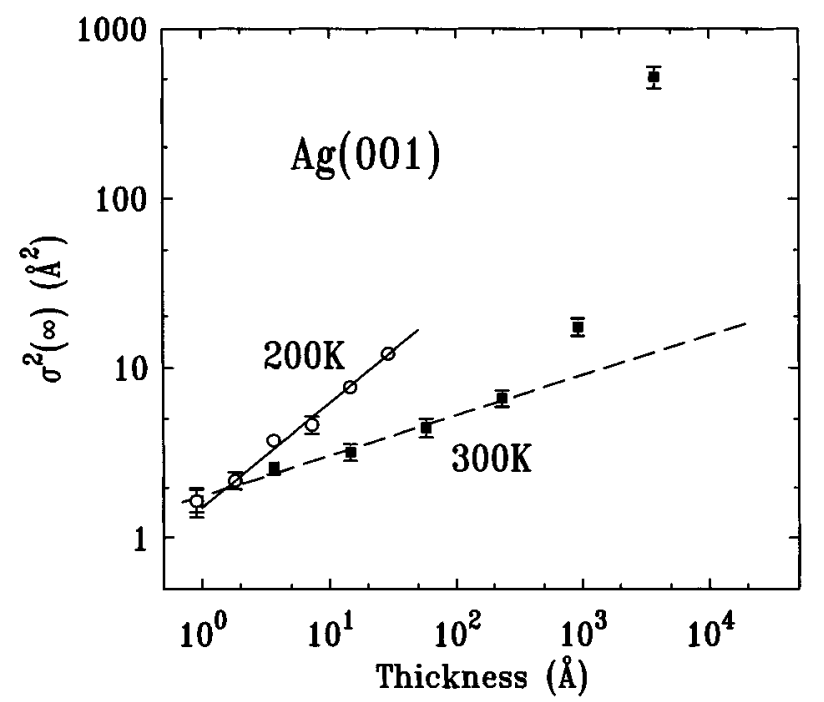

FIG. 5. The mean-square roughness of the $\operatorname{Ag}(001)$ surface is shown as a function of the deposited $\mathrm{Ag}$ thickness. The curves are obtained from a fit to $\sigma^{2} \propto t^{2 \beta}$, yielding $\beta=0.28 \pm 0.03$ at $200 \mathrm{~K}$ and $\beta=0.17 \pm 0.02$ at $300 \mathrm{~K}$. 
able theoretical evidence that $\beta=\frac{1}{2}$ is associated with a large step-ledge barrier.

We also point out that the roughening of the $\operatorname{Ag}(111)$ surface for $\beta \approx \frac{1}{2}$ does not arise from noise in the deposition flux. For a given deposited thickness $t$, the noise contribution to the roughness is substantially reduced from its maximum of $\sigma^{2}=d t$ because the height fluctuations come in increments of one atomic layer over lateral length-scales of a mean terrace size (which is many interatomic distances ${ }^{18,23}$ ). The roughness magnitude observed in our experiments at low temperature is much larger than what would be expected from the noise contribution; therefore, the roughening has a deterministic origin which is evidently related to the coarsening dynamics of mounds.

Another interesting feature of our data is that, for temperatures below $300 \mathrm{~K}$, the roughness magnitude decreases steadily with increasing temperature, despite the fact that $\beta \approx$ $\frac{1}{2}$ and does not change with temperature. This feature, along with the data in Figs. 2 and 3, bear a striking resemblance to computer simulations by Zhang, Detch, and Metiu, ${ }^{9}$ which address the effects of a step-ledge barrier. Although the simulations were performed in one dimension, these salient features should also hold in two dimensions. ${ }^{26}$ Unfortunately, very little can be found in the literature on the roughness magnitude for models which obtain $\beta \approx \frac{1}{2}$.

Previously, few experiments have quantitatively examined the temperature dependence of the roughness in detail. Our studies show that the roughness decreases with increasing temperature for both $\mathrm{Ag}(111)$ and $\mathrm{Ag}(001)$, in contrast to $\mathrm{Cu}(001)$ homoepitaxy ${ }^{10}$ which exhibits the opposite temperature dependence. There are several kinetic Monte Carlo simulations ${ }^{4,6,8,27}$ showing that either temperature dependence may occur (including reentrant growth ${ }^{4,14}$ ). These simulations generally include two mechanisms for surmounting a step ledge: thermal activation and some higher-order step-crossing process (kink sites, downward funneling, etc.) which is significant only at low temperature where the terrace sizes are small. Based on this we discuss $\operatorname{Ag}(001)$ and $\operatorname{Ag}(111)$. Below $300 \mathrm{~K}$ on the $\operatorname{Ag}(111)$ surface, thermal activation over the step-ledge barrier is negligible, and the high surface mobility on $\mathrm{Ag}(111)$ produces terrace sizes ${ }^{18}$ that are too large to permit significant low-temperature step-crossing processes, thus leading to $\beta=\frac{1}{2}$ in this limit. The transition to smaller $\beta$ above $300 \mathrm{~K}$ is evidently due to thermal activation over the step-ledge barrier, and $\beta$ appears to approach zero rapidly at high temperature as the system tends toward stepflow growth. ${ }^{17}$ For $\mathrm{Ag}(001)$ the $\beta$ values are significantly smaller than for $\operatorname{Ag}(111)$, and both the roughness magnitude as well as $\beta$ decrease with increasing temperature. This implies there is thermal activation over the step-ledge barrier for the measured temperature range, $T \geqslant 160 \mathrm{~K}$, consistent with the observation in Fig. 4 of intensity oscillations during deposition. Thus the additional energy barrier for diffusion over a step ledge on $\operatorname{Ag}(001)$ is significantly smaller than on $\mathrm{Ag}(111)$. Using a step-ledge barrier which is about five times smaller than on $\operatorname{Ag}(111)$, recent Monte Carlo simulations for $\mathrm{Ag}(001)$ by Zhang et al. $^{28}$ predict $\beta=0.25$ at $200 \mathrm{~K}$-in good agreement with our experimental results. A striking feature of the results for both crystal orientations is the substantial dependence on temperature- this is not fully appre-

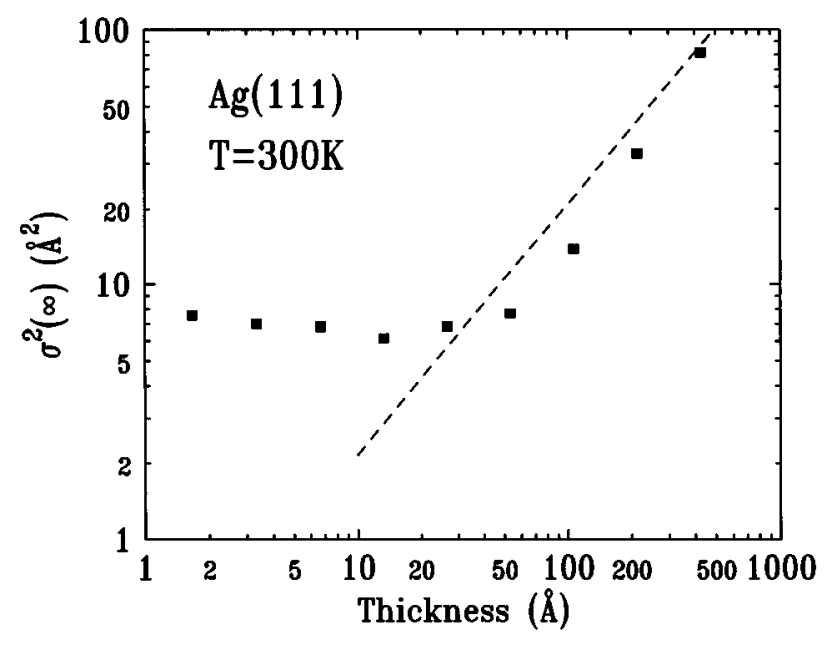

FIG. 6. Transient growth kinetics are observed when the surface is initially sputter roughened to $\sigma \approx 3 \AA$ before deposition. The dashed curve is the result obtained (from Fig. 2) for a smooth starting surface.

ciated from previous experimental studies which report power laws.

The existence of mounds during homoepitaxial growth is well established for $\operatorname{Ag}(111),{ }^{18}$ as well as for other systems such as $\mathrm{Fe}(001),{ }^{12} \mathrm{Cu}(001),{ }^{10} \mathrm{GaAs}(001),{ }^{11}$ and $\mathrm{Ge}(001) .{ }^{13}$ Although we measured the rms roughness which characterizes the vertical surface evolution, another approach has been to look only at the lateral coarsening of mounds. Experiments ${ }^{12}$ on $\mathrm{Fe}$ find that the mound separation coarsens as $\propto t^{n}$, with $n$ between 0.17 and 0.25 , consistent with a model by Siegert and Plischke ${ }^{7}$ (SP) which predicts $n=\frac{1}{4}$. SP originally suggested that the rms roughness evolves with the same exponent, i.e., $\beta=n=\frac{1}{4}$, which is in reasonable agreement with experiments on $\mathrm{Fe}(001)$. More recent theoretical work $^{27,29,30}$ shows that $\beta>n$ if the average mound slope does not saturate. $\operatorname{Ag}(111)$ would seem to fall into this category given the large value of $\beta$, as well as a slope which grows linearly with coverage in studies ${ }^{23}$ performed at low coverages. However, it is not clear whether significant growth in slope will persist over the nearly three decades in coverage for which we observe $\beta \approx \frac{1}{2}$. This brings two issues into focus: (1) the relationship between the vertical and lateral mound coarsening needs to be carefully addressed; and (2) there may be different types of mounds. We note that the step-ledge barrier relative to the surface diffusion barrier is much smaller for $\mathrm{Fe}(001)$ (Refs. 5 and 6) than for $\mathrm{Ag}(111),{ }^{19}$ so that mounds might behave quite differently in the two systems. Therefore, future experiments need to measure the lateral $(n)$ and vertical $(\beta)$ evolution during mound coarsening simultaneously.

Finally, we address the effects of an imperfect starting surface. Figure 6 shows the result of growing on a $\operatorname{Ag}(111)$ surface that was first roughened a small amount $(\sigma=3 \AA)$ by Ar-ion sputtering. With Ag deposition there is a slight initial smoothing, followed by an increasing roughness that approaches the result obtained from the smooth starting surface (dashed curve). Both the long-term stability of the roughened starting surface and the initial smoothing during deposition demonstrate the low rate for detachment of atoms from preexisting islands, and that only newly deposited monomers 
significantly contribute to the surface relaxation. The results in Fig. 6 suggest classic transient behavior: initially, growth is dominated by relaxation of the initial surface condition, whereas surface coursening occurs at late times and leads to a steady-state regime given by a power law. What is particularly striking is that transient effects are observable at thicknesses which are much larger than the initial roughness magnitude. Therefore, high-quality starting surfaces of the type depicted in Fig. 1 are critical for quantitative experiments.

In conclusion, we observe a pronounced temperature and orientation dependence of the rms roughness exponent $\beta$ for Ag homoepitaxy. This system offers both small and large step-ledge barriers, with $\operatorname{Ag}(111)$ representing the latter-it is the limiting case where the step-ledge barrier dominates the growth kinetics. Because the experiments examine a broad temperature and thickness range, these results provide important constraints for theoretical models of film growth which seek to incorporate multiple kinetic mechanisms.

\section{ACKNOWLEDGMENTS}

We thank M. Bartelt, J. Amar, J. Evans, F. Family, Z. Zhang, A. Zangwill, and S. Das Sarma for helpful discussions. Support is acknowledged from the University of Missouri Research Board, the NSF under Contract Nos. DMR9202528 and DMR-9623827, and the Midwest Superconductivity Consortium (MISCON) under DOE Grant No. DE-FG02-90ER45427. The SUNY X3 beamline is supported by the DOE under Contract No. DE-FG0286ER45231, and the NSLS is supported by the DOE, Div. of Materials Sciences and Div. of Chemical Sciences. One of us (W.C.E.) acknowledges support from the GAANN program of the U.S. Department of Education. We thank Ian Robinson for the $\operatorname{Ag}(111)$ crystal.
${ }^{1}$ See reviews: Dynamics of Fractal Surfaces, edited by F. Family and T. Vicsek (World Scientific, Singapore, 1991); A.-L. Barabási and H. E. Stanley, Fractal Concepts in Growth (Cambridge University Press, Cambridge, 1995); P. Meakin, Phys. Rep. 235, 189 (1993); J. Krug and H. Spohn, in Solids Far From Equilibrium, edited by C. Godrèche (Cambridge University Press, Cambridge, 1992).

${ }^{2}$ J. Chevrier, V. Le Thanh, R. Buys, and J. Derrien, Europhys. Lett. 16, 737 (1991); R. Chiarello, V. Panella, and J. Krim, Phys. Rev. Lett. 67, 3408 (1991); Y.-L. He, H.-N. Yang, T.-M. Lu, and G.-C. Wang, ibid. 69, 3770 (1992); J. Krim, I. Heyvaert, C. Van Haesendonck, and Y. Bruynseraede, ibid. 70, 57 (1993); H. You, R. P. Chiarello, H. K. Kim, and K. G. Vandervoort, ibid. 70, 2900 (1993); C. Thompson, G. Palasantzas, Y. P. Feng, S. K. Sinha, and J. Krim, Phys. Rev. B 49, 4902 (1994); G. Palasantzas and J. Krim, Phys. Rev. Lett. 73, 3564 (1994); W. M. Tong, R. S. Williams, A. Yanase, Y. Segawa, and M. S. Anderson, ibid. 72, 3374 (1994).

${ }^{3}$ J. Villain, J. Phys. (Paris) 1, 19 (1991).

${ }^{4}$ J. Jacobsen, K. W. Jacobsen, P. Stoltze, and J. K. Nørskov, Phys. Rev. Lett. 74, 2295 (1995).

${ }^{5}$ J. G. Amar and F. Family, Phys. Rev. B 52, 13081 (1995).

${ }^{6}$ M. C. Bartelt and J. W. Evans, Phys. Rev. Lett. 75, 4250 (1995).

${ }^{7}$ M. Siegert and M. Plischke, Phys. Rev. Lett. 73, 1517 (1994).

${ }^{8}$ P. Smilauer, M. R. Wilby, and D. D. Vvedensky, Phys. Rev. B 47, 4119 (1993).

${ }^{9}$ Z. Zhang, J. Detch, and H. Metiu, Phys. Rev. B 48, 4972 (1993).

${ }^{10}$ H.-J. Ernst, F. Fabre, R. Folkerts, and J. Lapujoulade, Phys. Rev. Lett. 72, 112 (1994).

${ }^{11}$ M. D. Johnson, C. Orme, A. W. Hunt, D. Graff, J. Sudijono, L. M. Sander, and B. G. Orr, Phys. Rev. Lett. 72, 116 (1994).

${ }^{12}$ J. A. Stroscio, D. T. Pierce, M. Stiles, and A. Zangwill, Phys. Rev. Lett. 75, 4246 (1995); K. Thürmer, R. Koch, M. Weber, and K. H. Rieder, ibid. 75, 1767 (1995).

${ }^{13}$ J. E. Van Nostrand, S. J. Chey, M.-A. Hasan, D. G. Cahill, and J. E. Greene, Phys. Rev. Lett. 74, 1127 (1995).

${ }^{14}$ R. Kunkel, B. Poelsema, L. K. Verheij, and G. Comsa, Phys. Rev. Lett. 65, 733 (1990); M. Bott, T. Michely, and G. Comsa, Surf. Sci. 272, 161 (1992).
${ }^{15}$ S. Das Sarma and S. V. Ghaisas, Phys. Rev. Lett. 69, 3762 (1992).

${ }^{16}$ A. S. Arrott, B. Heinrich, and S. T. Purcell, in Kinetics of Ordering and Growth at Surfaces, edited by M. G. Lagally (Plenum, New York, 1990), p. 321; J. Sudijono, M. D. Johnson, C. W. Snyder; M. B. Elowitz, and B. G. Orr, Phys. Rev. Lett. 69, 2811 (1992); H.-J. Ernst, F. Fabre, R. Folkerts, and J. Lapujoulade, J. Vac. Sci. Technol. A 12, 1809 (1994).

${ }^{17}$ H. A. van der Vegt, H. M. van Pinxteren, M. Lohmeier, E. Vlieg, and J. M. C. Thornton, Phys. Rev. Lett. 68, 3335 (1992).

${ }^{18}$ J. Vrijmoeth, H. A. van der Vegt, J. A. Meyer, E. Vlieg, and R. J. Behm, Phys. Rev. Lett. 72, 3843 (1994).

${ }^{19}$ K. Bromann, H. Brune, H. Röder, and K. Kern, Phys. Rev. Lett. 75, 667 (1995).

${ }^{20}$ W. F. Egelhoff and I. Jacob, Phys. Rev. Lett. 62, 921 (1989).

${ }^{21}$ P. Bedrossian, B. Poelsema, G. Rosenfeld, L. C. Jorritsma, N. N. Lipkin, and G. Comsa, Surf. Sci. 334, 1 (1995); Y. Suzuki, H. Kikuchi, and N. Koshizuku, Jpn. J. Appl. Phys. 27, L1175 (1988).

${ }^{22}$ W. C. Elliott, P. F. Miceli, T. Tse, and P. W. Stephens, Physica B 221, 65 (1996).

${ }^{23}$ Ch. Ammer, T. Schaefer, Ch. Teichert, K. Meinel, and M. Klaua, Surf. Sci. 307-309, 570 (1994).

${ }^{24}$ E. Z. Luo, J. Wollschläger, F. Wegner, and M. Henzler, Appl. Phys. A 60, 19 (1995).

${ }^{25}$ S. Das Sarma and P. Tamborenea, Phys. Rev. Lett. 66, 325 (1991).

${ }^{26} \mathrm{Z}$. Zhang (private communication).

${ }^{27}$ J. Amar and F. Family, Phys. Rev. B 54, 14071 (1996); F. Family and J. G. Amar, in Evolution of Epitaxial Structure and Morphology, edited by A. Zangwill, D. Jesson, D. Chambliss, and R. Clarke, MRS Symposia Proceedings No. 399 (Material Research Society, Pittsburgh, 1996), p. 67.

${ }^{28}$ C.-M. Zhang, M. C. Bartelt, J.-M. Wen, C. J. Jenks, J. W. Evans, and P. A. Thiel (unpublished).

${ }^{29}$ P. Smilauer and D. D. Vvedensky, Phys. Rev. B 52, 14263 (1995).

${ }^{30}$ L. Golubovic, in Evolution of Epitaxial Structure and Morphology, edited by A. Zangwill, D. Jesson, D. Chambliss, and R. Clarke, MRS Symposia Proceedings No. 399 (Materials Research Society, Pittsburgh, 1996), p. 257. 